\title{
Anterior and posterior segment vasculopathy associated with long-term use of tetrahydrozoline
}

\author{
E. Kisilevsky MD, D.D. DeAngelis MD
}

Cite as: CMAJ 2018 October 9;190:E1208. doi: 10.1503/cmaj.180519

A n otherwise healthy 45-year-old woman who has been using tetrahydrozoline hydrochloride in both eyes twice daily for 25 years presented to the ophthalmology clinic with worsening, chronic conjunctival corkscrew vessels bilaterally (Figure $1 \mathrm{~A}$ ). She reported no improvement since stopping tetrahydrozoline nine months earlier. On fundus examination, we found dilated, tortuous retinal vessels bilaterally (Figure 1B). The remainder of our ocular examination, including vision, intraocular pressure and extraocular motility, was normal. Assessment by a retinal specialist and fundus angiography were unremarkable. Examinations using magnetic resonance imaging and angiography of the brain and orbits were normal. Results for blood cell counts and thyroid testing were normal. At follow-up six months later, there was no change in the appearance of her eyes.

Tetrahydrozoline is an $\alpha$-adrenergic agonist that causes vasoconstriction and is used in over-the-counter ophthalmic decongestants. Prolonged use can cause tachyphylaxis and rebound hyperemia after stopping its use. ${ }^{1}$

Retinal vascular tortuosity can represent a normal variant, localized retinal vascular malformation and tumours, or systemic conditions such as diabetes, hypertension and hyperviscous states as found in multiple myeloma or Castleman disease. ${ }^{2,3}$

Dilation of conjunctival blood vessels is a common sign that can represent life-threatening conditions, such as carotid-cavernous fistulas or cavernous sinus thrombosis, or more benign conditions, such as allergic conjunctivitis or dry eye syndrome. For benign causes, lubrication of the ocular surface may be used for symptomatic relief. Patients often use ophthalmic decongestants interchangeably with lubrication, with the added effect of improved cosmesis.

We hypothesize that vascular remodelling, as seen in this patient, may be associated with vasoconstrictor-induced tissue

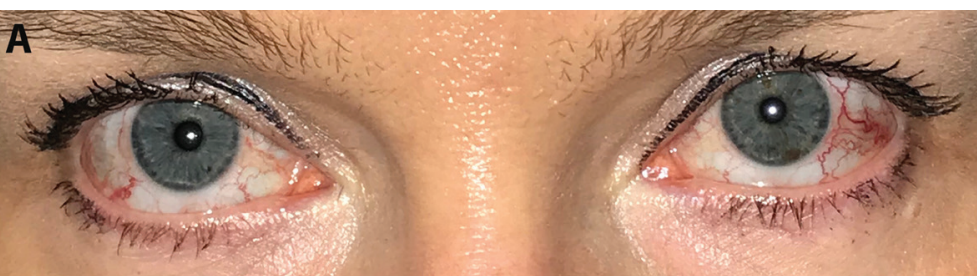

B

Figure 1: (A) Bilateral conjunctival corkscrew vessels in the eyes of a 45-year-old woman after long-term daily use of tetrahydrozoline. (B) Fundus examination of the right eye showing tortuous retinal arteries and veins with otherwise normal optic disc and retina.

\footnotetext{
Competing interests: None declared.

This article has been peer reviewed.

The authors have obtained patient consent.

Affiliations: Department of Ophthalmology and Vision Science (Kisilevsky, DeAngelis), University of Toronto; Mount Sinai Hospital (DeAngelis); Hospital for Sick Children (DeAngelis), Toronto, Ont.
}

ischemia, with subsequent release of vasodilating substances. Because ophthalmic decongestants are over-the-counter medications, their use has little direct medical oversight. Anterior and posterior segment vasculopathy associated with long-term use of tetrahydrozoline should be considered a potential adverse effect when physicians counsel patients about its use.

\section{References}

1. Soparkar CN, Wilhelmus KR, Koch DD, et al. Acute and chronic conjunctivitis due to over-the-counter ophthalmic decongestants. Arch Ophthalmol 1997;115:34-8.

2. Aung A, MacCumber MW. Castleman disease and retinal vascular tortuosity. JAMA Ophthalmol 2017;135:e164660.

3. Cheung CY, Zheng Y, Hsu W, et al. Retinal vascular tortuosity, blood pressure, and cardiovascular risk factors. Ophthalmology 2011;118:812-8.
Acknowledgement: The authors would like to thank Dr. Victoria Leung for her contribution in drafting the manuscript.

Correspondence to: Eli Kisilevsky, drkisilevsky@gmail.com 